\title{
Low temperature growth of clean single layer hexagonal boron nitride flakes and film for graphene- based field-effect transistors
}

\author{
Lifeng Wang ${ }^{1,2,3}$, Bin $\mathrm{Wu}^{{ }^{*}}$, Hongtao Liu ${ }^{1}$, Hanlin Wang ${ }^{1}$, Yuyu $\mathrm{Su}^{3}$, Weiwei Lei ${ }^{3}$, PingAn $\mathrm{Hu}^{2^{*}}$ and \\ Yunqi Liu ${ }^{1 *}$
}

Two dimensional (2D) materials have attracted intense attention owing to their unique physical and chemical properties and the realization of artificial structure and functionality [1]. Insulating hexagonal boron nitride (h$\mathrm{BN})$, a one-atom thick crystal with a large bandgap (6 eV) [2], superior chemical and thermal stability, and a higher thermal conductivity, is known as "white graphene" due to their similar structure-a lattice mismatch of only $1.6 \%$. The surface of $\mathrm{h}-\mathrm{BN}$ is chemically inert and lacks charge traps and dangling bonds compared with conventional $\mathrm{SiO}_{2}$ substrates [3-6]. h-BN films as dielectric layers with all of these properties enable integration with graphene for improving the performance of graphene devices [3-7]. In general, while the complete control over h-BN properties via single growth technique such as quality, crystallinity or layer number is difficult, controlling certain parameters of h-BN in growth process is allowed. In particular, the realization of low cost and high quality h-BN remains a great challenge at present.

Recently, several methods have been developed to grow monolayer or multi-layer h-BN film. In these methods, chemical vapor deposition (CVD) is very popular for obtaining high yield, better quality and controllable h-BN film on different metal substrates $(\mathrm{Cu}, \mathrm{Ni}, \mathrm{Pt})$ at growth temperature as high as $1,000^{\circ} \mathrm{C}$ [8-18]. While exfoliated $\mathrm{h}$-BN films are demonstrated to be a super dielectric layer for graphene devices, in practice, the performance of asgrown products as dielectric substrate of graphene fieldeffect transistor (FET) devices is dependent on the quality of $\mathrm{h}-\mathrm{BN}$ and interface properties between graphene and h-BN [8]. For industrial-level synthesis, the growth at low temperature is preferred for compatibility with the existing technology as energy consumption is reduced. Indeed, a method with low-energy consumption is highly desired for the synthesis of clean high-quality h-BN at low temperature. Plasma-enhanced CVD (PECVD) allows the low-temperature growth of h-BN on metals $[19,20]$, or even on dielectric surface [21,22]. The PECVD process, usually at $400^{\circ} \mathrm{C}$, is able to reduce energy cost and potentially useful for various applications in material fabrication industries. However, there are several challenges by this technique to grow h-BN film. On the one hand, amorphous state could be present in h-BN thin films produced by PECVD mainly due to the low preparation temperatures. On the other hand, ammonia borane molecules used as precursor are dissociated into three products: hydrogen, polyiminoborane $\left(\mathrm{BH}_{2} \mathrm{NH}_{2}\right.$, solid), and borazine $\left((\mathrm{HBNH})_{3}\right.$, gas; active species for h-BN growth) $[5,9,12-15]$, in which very active monomeric aminoborane forms polymeric aminoborane non-crystalline nanoparticles $(50-100 \mathrm{~nm})$ [12]. At present, there is no report overcoming these challenges.

Here we prepared high-quality triangle-shaped h-BN flakes and films on $\mathrm{Cu}$ surface via PECVD method at low temperature $\left(500^{\circ} \mathrm{C}\right)$, which is the lowest growth temperature reported to date via solid precursor (ammonia borane complex). The synthesized $\mathrm{h}-\mathrm{BN}$ flakes are regular triangular-shaped, monolayer and single crystalline by adjusting the growth parameters, such as the ratios of Ar to $\mathrm{H}_{2}$, plasma power and the distance between $\mathrm{Cu}$ foil and the reaction precursor. The side effect of aminoborane nanoparticles is eliminated by wrapping the precursor up

\footnotetext{
${ }^{1}$ Beijing National Laboratory for Molecular Sciences, Key Laboratory of Organic Solids, Institute of Chemistry, Chinese Academy of Sciences, Beijing 100190, China

${ }^{2}$ Key Lab of Microsystem and Microstructure, Ministry of Education, Harbin Institute of Technology, Harbin 150080, China

${ }^{3}$ Institute for Frontier Materials, Deakin University, Waurn Ponds, Victoria 3216, Australia

* Correspondence authors (emails: wubin@iccas.ac.cn (Wu B); hupa@hit.edu.cn (Hu P); liuyq@iccas.ac.cn (Liu Y))
} 
in tin foil. More importantly, graphene FET using the h$\mathrm{BN}$ films as the dielectric layers shows the highest hole and electron mobility of 10,500 and $4,750 \mathrm{~cm}^{2} \mathrm{~V}^{-1} \mathrm{~s}^{-1}$, respectively, which are higher than those using high temperature CVD $[4-7,10]$, demonstrating high quality of the as-grown h-BN.

We used solid ammonia borane as the source, which has equal number of boron and nitrogen, to explore h-BN growth on $\mathrm{Cu}$ surface by PECVD. A heating belt was used to heat the source, and $\mathrm{Ar}$ and $\mathrm{H}_{2}$ gases were used as carrier gas for flowing evaporated source molecules into the reaction chamber. Borazine molecules are produced at temperatures ranging between 90 and $110^{\circ} \mathrm{C}$, and they are active species for the h-BN growth on $\mathrm{Cu}$ (Fig. 1a). However, monomeric aminoborane is very active, and tends to aggregate to polymeric aminoborane particles that have poor crystallinity but stability at ambient conditions. Experimentally, we found that the triangular shaped h-BN flakes were generally covered with some white nanoparticles (Fig. S1), which were formed by the polymerization of active monomeric aminoborane carried to the h-BN surface by gas flow subjected to plasma bombardment. To reduce the amount of active monomeric aminoborane on $\mathrm{Cu}$ surface where h-BN flakes and films grow, we wrapped the precursor up in tin foil to prevent the diffusion of active monomeric aminoborane to the reaction zone. Fig. S2a, S2b show optical images of the tin foil surface before and after growth, respectively, where many h-BN nanoparticles are formed on tin surface after growth.

The synthesis of h-BN flakes and films on $\mathrm{Cu}$ surface was carried out using $\mathrm{Ar}$ and $\mathrm{H}_{2}$ as the carrier gas with 40 $\mathrm{sccm}$ and $20 \mathrm{sccm}$ flow rates (standard cubic centimeters per minute) at different growth temperatures (500, 550, 650 and $750^{\circ} \mathrm{C}$ ), a pressure of about $110-120 \mathrm{~Pa}$, and PECVD power of $70 \mathrm{~W}$. To tune the nucleation density of $\mathrm{h}-\mathrm{BN}, \mathrm{Cu}$ foils were annealed to reduce grain boundaries and roughness of $\mathrm{Cu}$ surface under $\mathrm{Ar} / \mathrm{H}_{2}$ atmosphere at $1,060^{\circ} \mathrm{C}$ for $6 \mathrm{~h}$. Fig. $1 \mathrm{~b}-\mathrm{e}$ show the scanning electron microscopy (SEM) images of $\mathrm{h}-\mathrm{BN}$ triangular flakes grown on annealed $\mathrm{Cu}$ foils at various growth temperatures, where the size distribution of h-BN flakes changed in the range of $1-2,2-3,3-4$, and $4-5 \mu \mathrm{m}$ with increasing growth temperatures. Fig. If shows h-BN size statistics analyzed from the corresponding SEM images for four examples, illustrating that h-BN nucleation density is reduced at high temperature associated with lower supersaturation and high thermal motion of precursors [5,23]. In addition, the estimated growth rates for each case essentially remain unchanged (about 1-1.5
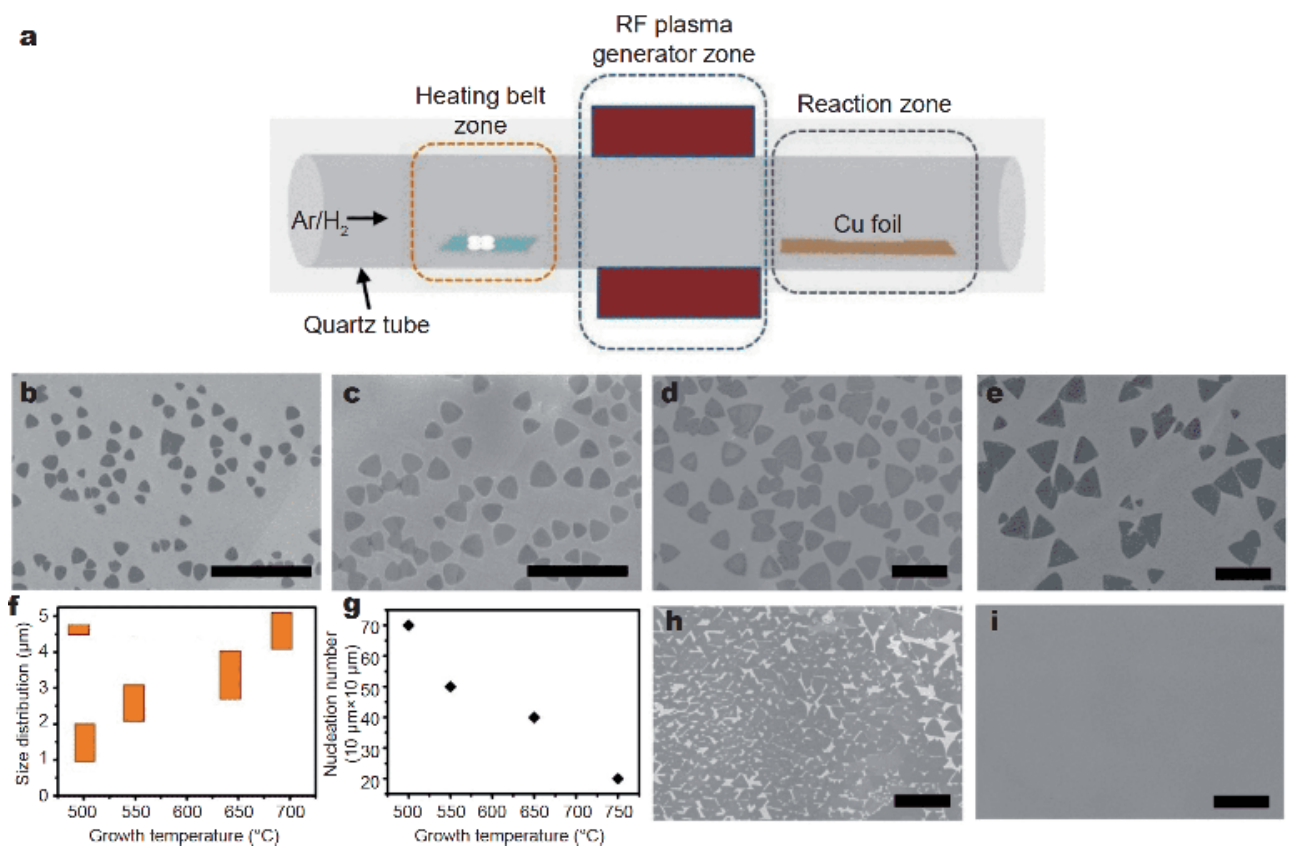

Figure 1 (a) The schematic showing remote radiofrequency (RF) PECVD setup. (b-e) SEM images of h-BN synthesized at different temperatures $\left(500,550,650\right.$ and $750^{\circ} \mathrm{C}$, respectively). The rest growth parameters are same: using Ar and $\mathrm{H}_{2}$ as the carrier gas with a flow rate of about 40 and $20 \mathrm{sccm}$, a pressure of about 110-120 Pa, and PECVD power of $70 \mathrm{~W}$. (f, g) The size distribution and nucleation number as a function of growth temperature. (h, i) SEM images showing that h-BN domains grow into near-fully covered and continuous film for longer growth time of 65 and $70 \mathrm{~min}$, respectively. All scale bars are $10 \mu \mathrm{m}$. 
$\mu \mathrm{m} \min ^{-1}$ ), which is $1-1.5$ times faster than those for CVD grown $\mathrm{h}-\mathrm{BN}$ at high growth temperature $\left(1,000^{\circ} \mathrm{C}\right)$ [5]. This result is reasonable as PECVD enables fast dissociation and dehydrogenation rates of precursors compared with conventional thermal CVD. The formation of continuous h-BN film is allowed in this technique, and the increase of growth duration yields gradual merging of as-synthesized h-BN (Fig. 1h), eventually leading to a continuous film without any gap (Fig. 1i).

Fig. 2a and $\mathrm{b}$ show the X-ray photoemission spectroscopy (XPS) spectra of the as-grown h-BN on $\mathrm{Cu}$ foil at $750^{\circ} \mathrm{C}$. The observed $\mathrm{B} 1 \mathrm{~s}$ and $\mathrm{N} 1 \mathrm{~s}$ binding energies are peaked at 190.37 and $397.97 \mathrm{eV}$, respectively (Fig. 2a, b), corresponding to the previously-reported locations of $h$ $\mathrm{BN}$ products $[5,8-18]$. The atomic ratio of $\mathrm{B} / \mathrm{N}$ analyzed from the XPS data is about 1.10 , approaching $1 / 1$ stoichiometry in h-BN. As shown in Fig. S3a-c, the XPS spectra of h-BN films with different grown temperatures $\left(500,550\right.$ and $\left.650^{\circ} \mathrm{C}\right)$ are similar to the spectrum shown in Fig. 2a, indicating that the configuration for $\mathrm{B}$ and $\mathrm{N}$ atoms of all grown samples adopts the hexagonal phase $\mathrm{B}-\mathrm{N}$ bonds. To investigate the characteristics of monolayer h-BN films at different growth temperatures, transparent quartz substrates were used as target substrates for the h-BN transfer, and then the samples were characterized by optical absorption. Fig. S4a shows a typical UV-visible absorption spectrum of as-grown monolayer h-BN. h-BN film has approximately no absorbance within the visible-light range, but a large absorption within the UV range, indicating an optical band
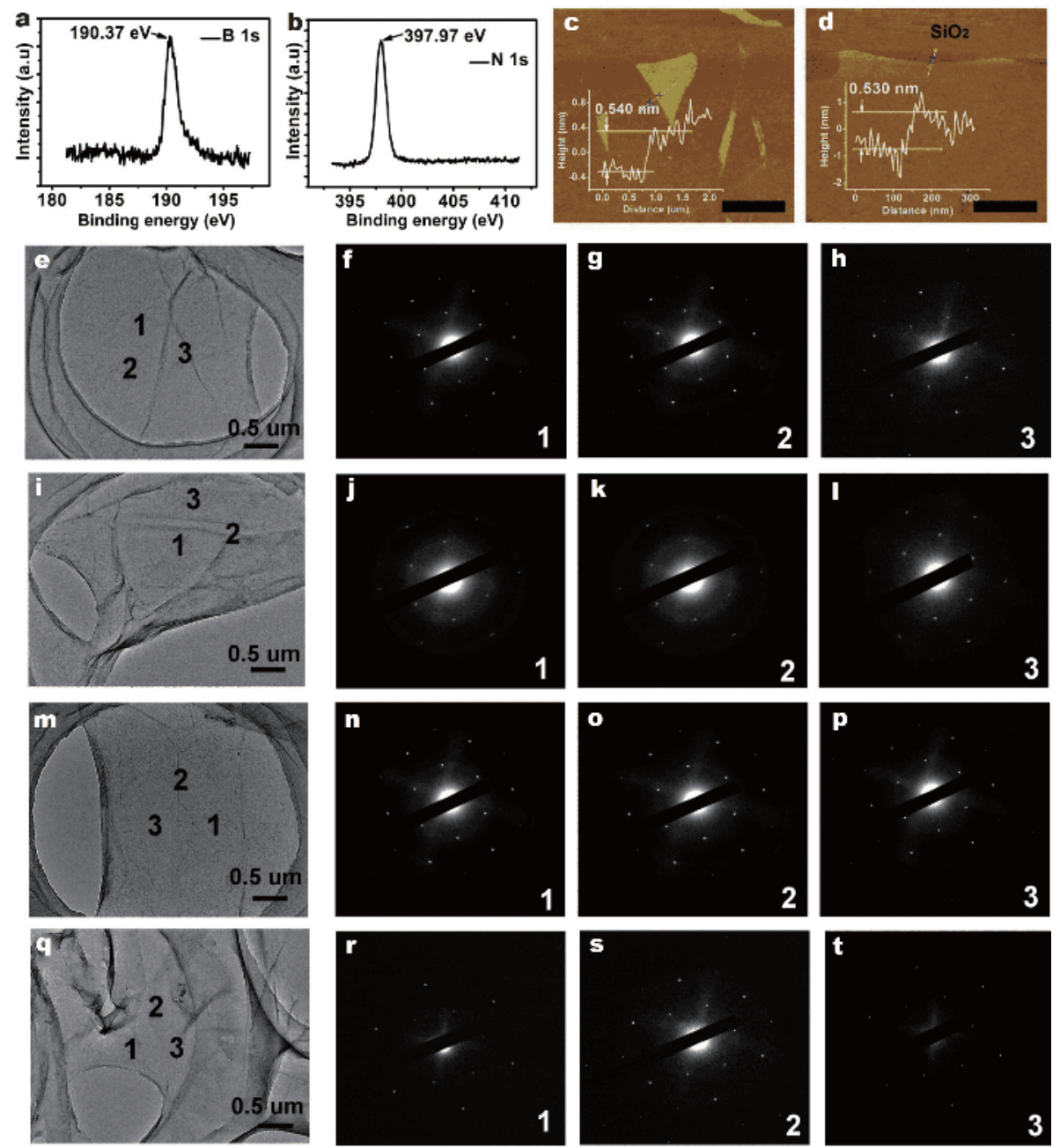

Figure 2 (a, b) XPS spectra of B 1s and $\mathrm{N}$ 1s for the transferred h-BN samples, respectively. (c, d) Typical AFM images of h-BN flake and film transferred onto $\mathrm{SiO}_{2} / \mathrm{Si}$ substrates by PMMA-assisted method, respectively. Insets in each image also show the height of h-BN grain along the labeled lines. All scale bars are $2 \mu \mathrm{m}$. (e, i, m and q) Typical TEM images of continuous h-BN films synthesized at 500, 550,650 and $750^{\circ} \mathrm{C}$, respectively. (f-h, $j-1, n-p$ and $r-t$ ) SAED patterns recorded on different locations labeled in image of e, i, $m$ and $q$, respectively. 
gap (OBG). OBG is determined to be around $6.04 \mathrm{eV}$ in Fig. S4b $[14,24]$. The slightly larger value of OBG compared with that of multi-layer or bulk h-BN resulted from the lacking of layer-layer interaction for single layer h-BN [25]. Indeed, our value is in good agreement with that of monolayer $\mathrm{h}-\mathrm{BN}$ obtained by the theoretical calculation $(6.0 \mathrm{eV})[26]$ and previous experiments $[5,9,12,15]$.

The transferred h-BN flakes and films on $\mathrm{SiO}_{2} / \mathrm{Si}$ substrates were characterized by atomic force microscopy (AFM) (Fig. 2c and d). Fig. S5a and S5b show the SEM images of triangular h-BN flakes and films transferred onto the $\mathrm{SiO}_{2} / \mathrm{Si}$ substrate, respectively. According to the previous report [5], we annealed the transferred h-BN samples at $950^{\circ} \mathrm{C}$ for $25 \mathrm{~min}$ to get rid of PMMA residues on h-BN surface in air. The treated h-BN flakes and films are about 0.540 and $0.530 \mathrm{~nm}$ in height (inset), which are in good agreement with the values obtained for monolayer h-BN $[5,12,15]$. The Raman spectrum of the h-BN film on the $\mathrm{SiO}_{2} / \mathrm{Si}$ substrate shows a peak at $1,369 \mathrm{~cm}^{-1}$, originating from the $\mathrm{B}-\mathrm{N}$ bond stretching of the $\mathrm{BN}$ films (Fig. S6).

Transmission electron microscopy (TEM) was further used to characterize the crystalline structure of the asgrown h-BN film at different growth temperatures (500, 550, 650 and $750^{\circ} \mathrm{C}$ ). Fig. 2e-t show a series of TEM images of the h-BN films grown with different temperatures transferred on the TEM grid along with selected area electron diffraction (SAED) patterns obtained at various locations. As shown in Fig. 2e-h, the film has a uniform contrast in Fig. 2e, and the recorded SAED spots exhibit a 6-fold symmetry that is characteristic of h-BN, suggesting its good crystallinity. Meanwhile, SAED spots obtained at various positions in an specific region of film exhibit the similar orientation (Fig. 2f-h), and two sets of diffraction patterns were also observed at marked position as shown in Fig. $2 \mathrm{~h}$, indicating that the h-BN film consists of small domains with various orientations due to small size in the individual flake. However, for the growth temperatures of $650^{\circ} \mathrm{C}$ and $750^{\circ} \mathrm{C}$, the synthesized h-BN film with large domains only shows one set of sixfold symmetric SAED pattern at different locations of the films with same orientation, demonstrating that it is single crystal. The result reflects that h-BN grains are continuously growing as a function of time.

To understand h-BN growth by PECVD, we investigated the effects of various critical parameters on the h-BN growth. Here we discuss the ratios of $\mathrm{Ar} / \mathrm{H}_{2}$ flow, plasma powers, growth temperature, growth duration, and the distance between growth zone and the plasma generator. Accordingly, carrier gas composition is sig- nificant to h-BN morphology. When $\mathrm{Ar} / \mathrm{H}_{2}$ flows (40/0 and $40 / 10 \mathrm{sccm}$ ) were used in the growth stage, random shaped objects in Fig. $3 \mathrm{a}$ and $3 \mathrm{~b}$ were obtained. Changing the $\mathrm{Ar} / \mathrm{H}_{2}$ flow to $40 / 20 \mathrm{sccm}$ resulted in the formation of h-BN flakes with triangular shape (Fig. 3c).

Fig. 3d-f display typical SEM images of h-BN flakes grown at different powers of plasma of 50,70 and $90 \mathrm{~W}$ in $\mathrm{Ar} / \mathrm{H}_{2}(40 / 20 \mathrm{sccm})$ gas flow, respectively. The average grain size is about $1 \mu \mathrm{m}$ at the condition of $50 \mathrm{~W}$ plasma, and the size is about $4 \mu \mathrm{m}$ using $70 \mathrm{~W}$ plasma. The size is about $0.8 \mu \mathrm{m}$ using $90 \mathrm{~W}$ plasma. The plots of h-BN size and the density of nucleation $v s$. plasma power in Fig. S7a, b display that a maximum size is grown at $70 \mathrm{~W}$ and is reduced to $2-3 \mu \mathrm{m}$ at a power higher than $90 \mathrm{~W}$. This result probably originates from a high concentration of hydrogen species produced at a high plasma power. Here, the hydrogen plays two roles in h-BN growth: an activator and an etching reagent [27]. Furthermore, we found the distance between growth zone and plasma generator obviously influences the synthesized h-BN. When the distance is about 20 and $25 \mathrm{~cm}$, we observed the h-BN flakes with flower-like morphology due to the effective etching (Fig. 3g, h), which is similar to the graphene etching [28]. To decrease the influence of etching, we moved the plasma generator away the growth zone to $35 \mathrm{~cm}$, and then regular and no-etched h-BN flakes were synthesized as shown in Fig. 3i.

In addition, we fabricated FET devices of monolayer graphene domains grown on liquid $\mathrm{Cu}$ surface $[29,30]$ using oxidation-cleaned h-BN monolayer films synthesized at different growth temperatures as back-gated and top-gated dielectrics in Fig. 4a, respectively. Briefly, single-layered h-BN film was transferred onto $300 \mathrm{~nm} \mathrm{SiO}{ }_{2} /$ Si substrate and oxidized in air, followed by transferring graphene flakes onto h-BN films. Device fabrication protocols were based on the previous reports $[29,30]$, and electrical transport measurements were conducted in ambient condition. Fig. $4 \mathrm{~b}$ and Fig. S8a-d show a series of optical images of graphene/h-BN films synthesized at different growth temperatures for FET devices. We tested more than 30 devices, and compared the difference of two type models in electrical properties. Compared with $\mathrm{SiO}_{2}$ substrates, the clean h-BN films for dielectrics exhibit the improved carrier mobility of graphene and the shift of Dirac point towards lower voltage. Fig. $4 \mathrm{c}$ shows typical transfer curves for h-BN films used as back-gated dielectric layers. The devices show typical p-type characteristic in agreement with our previous results of graphene/h-BN devices [5]. The shifting of Dirac points for four cases using h-BN as back-gated dielectric layers 

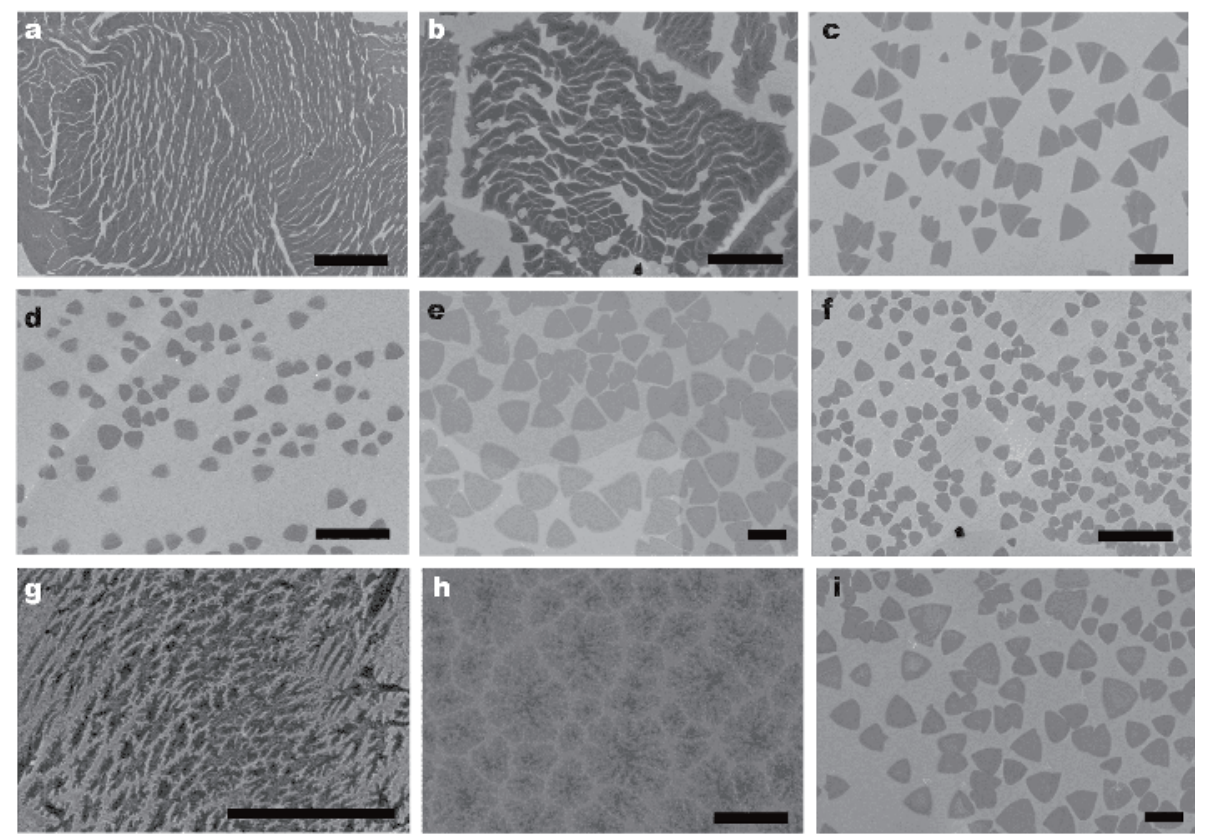

Figure 3 (a-c) SEM images of triangular-shaped h-BN domains grown on Cu foil surface with different ratio of gas flow $\left(\mathrm{Ar} / \mathrm{H}_{2}=40 / 0,40 / 10\right.$ and $40 /$ $20 \mathrm{sccm})$, plasma power $(70 \mathrm{~W})$ and the distance between $\mathrm{Cu}$ foil and plasma generator $(35 \mathrm{~cm}) .(\mathrm{d}-\mathrm{f})$ SEM images showing the as-grown triangularshaped h-BN domains with different plasma power (50, 70 and $90 \mathrm{~W}$, respectively), gas ratio $(40 / 20 \mathrm{sccm})$ and the distance between Cu foil and plasma generator $(35 \mathrm{~cm})$. (g-i) SEM images of the as-grown triangular-shaped h-BN domains with different distances between Cu foil and the plasma generator $(20,25$ and $35 \mathrm{~cm}$, respectively), gas ratio $(40 / 20 \mathrm{sccm})$ and plasma power $(70 \mathrm{~W})$. All scale bars are $5 \mu \mathrm{m}$.

can be clearly seen in Fig. $4 \mathrm{c}$, with $V_{\mathrm{g}}=12,5,10$ and $12 \mathrm{~V}$ for h-BN films synthesized at different temperatures (500, 550,650 and $750^{\circ} \mathrm{C}$ ), respectively. This is tunable by including graphene on $\mathrm{SiO}_{2} / \mathrm{Si}$ substrates as its Dirac point usually shifts to a much positive value with similar protocol for the device fabrication and device tests [28]. The shifting of Dirac points for graphene devices reflects graphene doping by substrates or environment. Indeed, the graphene devices on cleaned h-BN films grown at different temperatures show higher hole mobility than that on $\mathrm{SiO}_{2} / \mathrm{Si}$ substrates. For instance, hole carrier mobility is about 1,515, 974, 2,356, 3,321 and $10,500 \mathrm{~cm}^{2} \mathrm{~V}^{-1} \mathrm{~s}^{-1}$ at $V_{\mathrm{ds}}=0.01 \mathrm{~V}$ for $\mathrm{SiO}_{2}$ substrate, h-BN films synthesized at $500,550,650$ and $750^{\circ} \mathrm{C}$, respectively (Fig. S9). The hole carrier mobility of the devices using the low temperature $\mathrm{h}-\mathrm{BN}$ as dielectric layers is lower than that on the $\mathrm{SiO}_{2}$ substrates due to the $\mathrm{H}_{2} \mathrm{O}$ or other residue. The calculated electron carrier mobility for h-BN films synthesized at $500,550,650,750^{\circ} \mathrm{C}$, respectively, is about $302,392,1,162$ and $4,750 \mathrm{~cm}^{2} \mathrm{~V}^{-1} \mathrm{~s}^{-1}$ at $V_{\mathrm{ds}}=0.01 \mathrm{~V}$ as shown in Fig. S9. The mobility of different devices is improved by h-BN films as back-gated dielectrics grown at a higher temperature, due to the fact that the high temperature results in high crystallinity of h-BN. These results indicate that the quality of substrate is vital for improving the carrier mobility of graphene devices.

To prevent the degradation of graphene devices from the influence of water or gases in the air, we transferred the h-BN synthesized at $750^{\circ} \mathrm{C}$ to the post-tested devices as the top dielectric layer and the transferred process were similar to the previous report [5]. According to the tested results (Fig. 4e and f), we found the Dirac point of graphene devices with $\mathrm{h}-\mathrm{BN}$ as top-gated dielectric layers upshifted to $V_{\mathrm{g}}=40$ and $32 \mathrm{~V}\left(650\right.$ and $\left.750^{\circ} \mathrm{C}\right)$, respectively, due to the trapped water in the transfer at the h$\mathrm{BN} / g r a p h e n e$ interface. Owing to the high temperature stability of h-BN in air, the h-BN/graphene/h- $\mathrm{BN} / \mathrm{SiO}_{2} / \mathrm{Si}$ devices were annealed at $80^{\circ} \mathrm{C}$ in vacuum for 3 and $24 \mathrm{~h}$ to remove water residues due to the fabricated process. As shown in Fig. $4 \mathrm{e}$ and $\mathrm{f}$, the Dirac point of the graphene devices with h-BN as top-gated dielectric downshifted to $V_{\mathrm{g}}=38$ and $20 \mathrm{~V}\left(650\right.$ and $\left.750^{\circ} \mathrm{C}\right)$, respectively, but all these values are higher than those values in Fig. $4 \mathrm{c}$ due to less water residue. Meanwhile, the hole carrier mobility for the un-annealed devices in vacuum is $1,545,3,321$ and $4,000 \mathrm{~cm}^{2} \mathrm{~V}^{-1} \mathrm{~s}^{-1}$ at $V_{\mathrm{ds}}=0.01 \mathrm{~V}\left(\mathrm{SiO}_{2} / \mathrm{Si}\right.$ substrate, 650 and $750^{\circ} \mathrm{C}$ ), respectively (Fig. $4 \mathrm{~g}-\mathrm{i}$ ). While annealed for 3 and $12 \mathrm{~h}$ in vacuum, the hole carrier mobility is 2,863 and $2,829\left(650^{\circ} \mathrm{C}\right), 5,500 \mathrm{~cm}^{2} \mathrm{~V}^{-1} \mathrm{~s}^{-1}\left(750^{\circ} \mathrm{C}\right)$ at $V_{\mathrm{ds}}=0.01 \mathrm{~V}$. The calculated electron carrier mobility is about 1,261 
$\mathbf{a}$
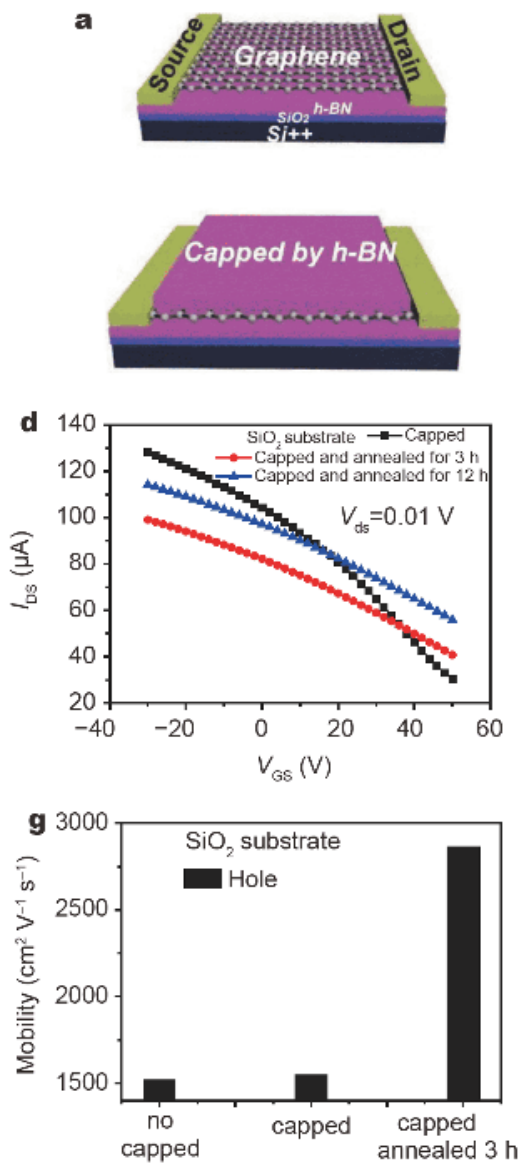

b
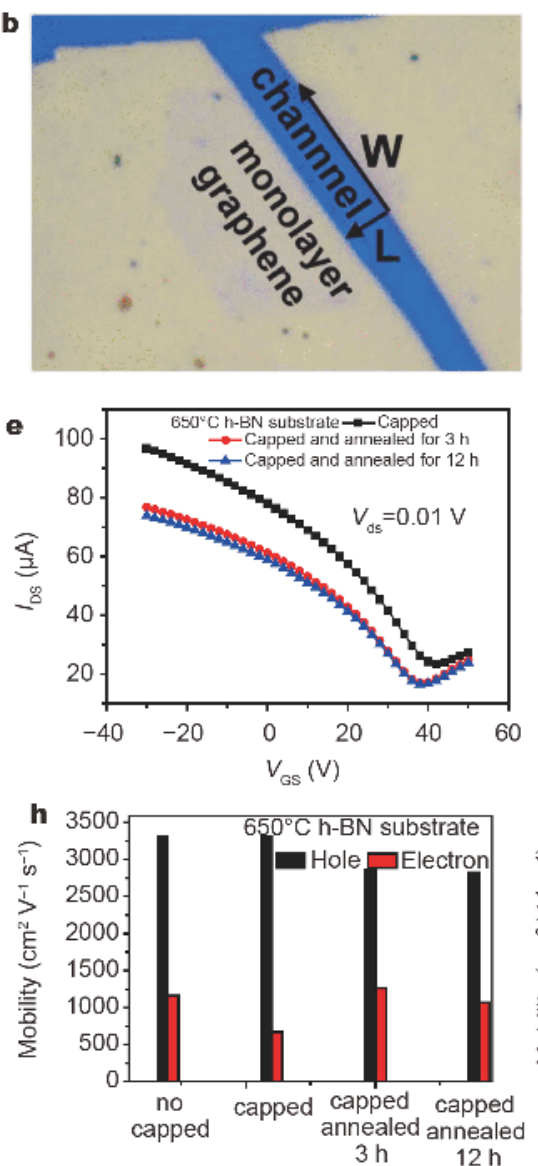
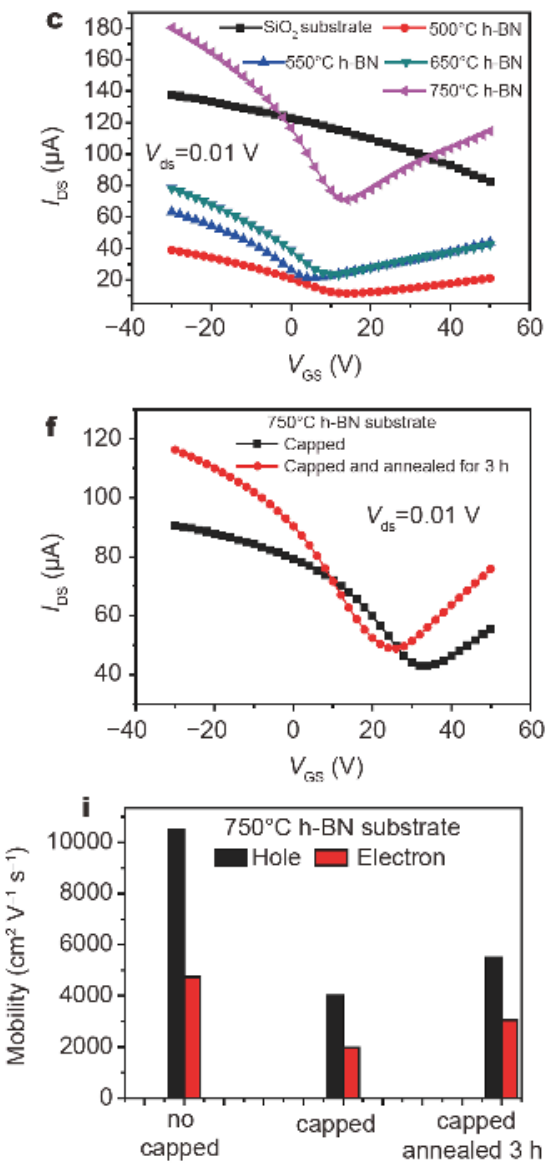

Figure 4 (a) The schematic diagram of graphene FET devices with h-BN as dielectrics: (1) h-BN as back gate dielectric; (2) h-BN as back gate and top gate dielectric. (b) The optical image of the device with h-BN dielectric grown at $750^{\circ} \mathrm{C}$. (c) Transfer curves of graphene/SiO $\mathrm{O}_{2}$ and graphene/h- $\mathrm{BN}$ films synthesized at different temperatures. (d) Transfer curves of graphene $/ \mathrm{SiO}_{2}$ capped by h-BN films synthesized at $750^{\circ} \mathrm{C}$ as the top gate dielectric and annealed in vacuum at $80^{\circ} \mathrm{C}$ for 3 and $12 \mathrm{~h}$. (e) Transfer curves of graphene/h-BN synthesized at $650^{\circ} \mathrm{C}$ capped by h-BN films synthesized at $750^{\circ} \mathrm{C}$ as the top gate dielectric and annealed in vacuum ambient at $80^{\circ} \mathrm{C}$ for 3 and $12 \mathrm{~h}$. (f) Transfer curves of graphene/h-BN synthesized at $750^{\circ} \mathrm{C}$ capped by h-BN films synthesized at $750^{\circ} \mathrm{C}$ as the top gate dielectric and annealed in vacuum ambient at $80^{\circ} \mathrm{C}$ for $3 \mathrm{~h}$. (g-i) Histograms of hole and electron mobility distribution for $\mathrm{SiO}_{2}$ and h-BN synthesized at different temperatures $\left(650\right.$ and $\left.750^{\circ} \mathrm{C}\right)$ as back- and top-gated dielectrics.

and $1,066\left(650^{\circ} \mathrm{C}\right), 3,064 \mathrm{~cm}^{2} \mathrm{~V}^{-1} \mathrm{~s}^{-1}\left(750^{\circ} \mathrm{C}\right)$ at $V_{\mathrm{ds}}=$ $0.01 \mathrm{~V}$, respectively. This trend is very important for improving the performance of the devices through decreasing the doping water in the air.

In summary, the high quality h-BN grew on $\mathrm{Cu}$ surface via PECVD method with low growth temperature and neat surface. Moreover, we elucidate that the quality of as-synthesized h-BN is modulated by growth temperature, and as dielectrics of graphene FET, the quality of asgrown $\mathrm{h}-\mathrm{BN}$ is vital for the device performance.

\section{Received 4 February 2019; accepted 16 March 2019;} published online 17 April 2019

1 Geim AK, Grigorieva IV. van der Waals heterostructures. Nature, 2013, 499: 419-425
2 Watanabe K, Taniguchi T, Kanda H. Direct-bandgap properties and evidence for ultraviolet lasing of hexagonal boron nitride single crystal. Nat Mater, 2004, 3: 404-409

3 Dean CR, Young AF, Meric I, et al. Boron nitride substrates for high-quality graphene electronics. Nat Nanotechnol, 2010, 5: 722726

4 Lee KH, Shin HJ, Lee J, et al. Large-scale synthesis of high-quality hexagonal boron nitride nanosheets for large-area graphene electronics. Nano Lett, 2012, 12: 714-718

5 Wang L, Wu B, Chen J, et al. Monolayer hexagonal boron nitride films with large domain size and clean interface for enhancing the mobility of graphene-based field-effect transistors. Adv Mater, 2014, 26: 1559-1564

6 Yang W, Chen G, Shi Z, et al. Epitaxial growth of single-domain graphene on hexagonal boron nitride. Nat Mater, 2013, 12: 792797

7 Lu G, Wu T, Yuan Q, et al. Synthesis of large single-crystal hexagonal boron nitride grains on $\mathrm{Cu}-\mathrm{Ni}$ alloy. Nat Commun, 2015, 6: 
6160

8 Zhang C, Fu L, Zhao S, et al. Controllable Co-segregation synthesis of wafer-scale hexagonal boron nitride thin films. Adv Mater, 2014, 26: 1776-1781

9 Kim G, Jang AR, Jeong HY, et al. Growth of high-crystalline, single-layer hexagonal boron nitride on recyclable platinum foil. Nano Lett, 2013, 13: 1834-1839

10 Wang M, Jang SK, Jang WJ, et al. A platform for large-scale graphene electronics-CVD growth of single-layer graphene on CVDgrown hexagonal boron nitride. Adv Mater, 2013, 25: 2746-2752

11 Shi Y, Hamsen C, Jia X, et al. Synthesis of few-layer hexagonal boron nitride thin film by chemical vapor deposition. Nano Lett, 2010, 10: 4134-4139

12 Wang L, Wu B, Liu H, et al. Water-assisted growth of large-sized single crystal hexagonal boron nitride grains. Mater Chem Front, 2017, 1: 1836-1840

13 Tay RY, Wang X, Tsang SH, et al. A systematic study of the atmospheric pressure growth of large-area hexagonal crystalline boron nitride film. J Mater Chem C, 2014, 2: 1650

14 Auwärter W, Suter HU, Sachdev H, et al. Synthesis of one monolayer of hexagonal boron nitride on $\mathrm{Ni}(111)$ from B-trichloroborazine $(\mathrm{ClBNH})_{3}$. Chem Mater, 2004, 16: 343-345

15 Gao Y, Ren W, Ma T, et al. Repeated and controlled growth of monolayer, bilayer and few-layer hexagonal boron nitride on $\mathrm{Pt}$ foils. ACS Nano, 2013, 7: 5199-5206

16 Ismach A, Chou H, Ferrer DA, et al. Toward the controlled synthesis of hexagonal boron nitride films. ACS Nano, 2012, 6: 6378-6385

17 Behura S, Nguyen P, Debbarma R, et al. Chemical interactionguided, metal-free growth of large-area hexagonal boron nitride on silicon-based substrates. ACS Nano, 2017, 11: 4985-4994

18 Tay RY, Griep MH, Mallick G, et al. Growth of large single-crystalline two-dimensional boron nitride hexagons on electropolished copper. Nano Lett, 2014, 14: 839-846

19 Vilcarromero J, Carreño MNP, Pereyra I. Mechanical properties of boron nitride thin films obtained by RF-PECVD at low temperatures. Thin Solid Films, 2000, 373: 273-276

20 Deb B, Bhattacharjee B, Ganguli A, et al. Boron nitride films synthesized by RF plasma CVD of borane-ammonia and nitrogen. Mater Chem Phys, 2002, 76: 130-136

21 Bath A, van der Put PJ, Becht JGM, et al. Plasma enhanced chemical vapor deposition and characterization of boron nitride gate insulators on InP. J Appl Phys, 1991, 70: 4366-4370

22 Zhang K, Yap FL, Li K, et al. Large scale graphene/hexagonal boron nitride heterostructure for tunable plasmonics. Adv Funct Mater, 2014, 24: 731-738

$23 \mathrm{Wu}$ B, Geng D, Guo Y, et al. Equiangular hexagon-shape-controlled synthesis of graphene on copper surface. Adv Mater, 2011, 23: 3522-3525

24 Yuzuriha TH, Hess DW. Structural and optical properties of plasma-deposited boron nitride films. Thin Solid Films, 1986, 140: 199-207

25 Hoffman DM, Doll GL, Eklund PC. Optical properties of pyrolytic boron nitride in the energy range 0.05-10 eV. Phys Rev B, 1984, 30: 6051-6056

26 Blase X, Rubio A, Louie SG, et al. Quasiparticle band structure of bulk hexagonal boron nitride and related systems. Phys Rev B, 1995, 51: 6868-6875

27 Vlassiouk I, Regmi M, Fulvio P, et al. Role of hydrogen in chemical vapor deposition growth of large single-crystal graphene. ACS
Nano, 2011, 5: 6069-6076

28 Geng D, Wu B, Guo Y, et al. Fractal etching of graphene. J Am Chem Soc, 2013, 135: 6431-6434

29 Geng D, Wu B, Guo Y, et al. Uniform hexagonal graphene flakes and films grown on liquid copper surface. Proc Natl Acad Sci USA, 2012, 109: 7992-7996

$30 \mathrm{Wu}$ B, Geng D, Xu Z, et al. Self-organized graphene crystal patterns. NPG Asia Mater, 2013, 5: e36

Acknowledgements This work was supported by the National Basic Research Program of China (2016YFA0200101), the National Natural Science Foundation of China (21633012 and 61890940), Beijing Municipal Science \& Technology Commission (Z161100002116025), Chinese Academy of Sciences, the Strategic Priority Research Program of the Chinese Academy of Sciences (XDB30000000 and XDB12030100) and Beijing National Laboratory for Molecular Sciences (BNLMS).

Author contributions All authors listed have made a substantial, direct and intellectual contribution to the work, and approved it for publication.

Conflict of interest The authors declare no conflict of interest.

Supplementary information The supplementary data are available in the online version of the paper.

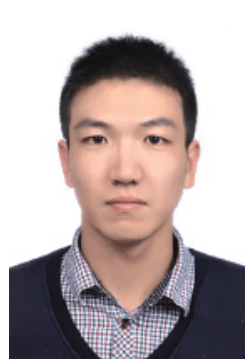

Lifeng Wang received his BE degree from Nanjing Tech University in 2011. He obtained his PhD degree from Harbin Institute of Technology in 2016 under the direction of Prof. Yunqi Liu (ICCAS) and Prof. PingAn Hu. Currently, he is a postdoctoral research fellow at Deakin University. His research interests focus on the preparation and application of $2 \mathrm{D}$ materials in electrical devices and energy storage and conversation.

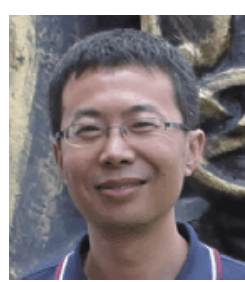

Bin Wu obtained his BSc degree in 1994 from Inner Mongolia University, China and his $\mathrm{PhD}$ degree in physical chemistry from Peking University in 2002. He joined the ICCAS as an associate professor in 2008 after doing postdoctoral research for several years. Now he is a professor at ICCAS. His research interests include the controlled growth of $2 \mathrm{D}$ materials and their electronic applications.

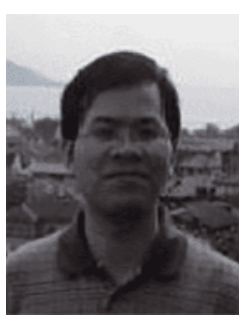

PingAn $\mathbf{H u}$ is a professor at Harbin Institute of Technology (HIT). He obtained his $\mathrm{PhD}$ degree from ICCAS. He got New Century Excellent Talents in University of China in 2010 and Longjiang Special Professorship in 2011. He published more than 100 SCI papers in highly qualified international journals. 


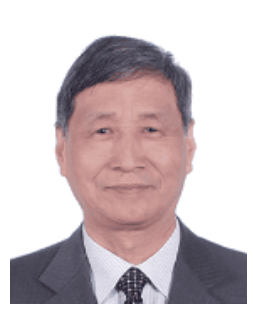

Yunqi Liu is a professor at ICCAS. He graduated from Nanjing University in 1975, and received a doctorate from Tokyo Institute of Technology, Japan, in 1991. He was selected as an Academician of CAS in 2015, and a member of the World Academy of Sciences in 2018. His research interests include molecular materials and devices, the synthesis and applications of carbon nanomaterials, and organic electronics. He has published more than 600 peer reviewed papers with over 30,000 citations.

低温制备高质量六方氮化嗍晶畴、薄膜及其在石 墨烯基场效应晶体管中的应用

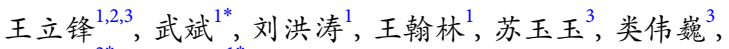
胡平安 ${ }^{2^{*}}$, 刘云折 ${ }^{1 *}$

摘要 二维六方氮化硼是一种理想的石墨烯电学器件介电层材料, 然而, 制备低成本和高质量的氮化嗍材料仍是一个挑战. 本文使用 等离子体化学气相沉积法, $500^{\circ} \mathrm{C}$ 下在铜䇴衬底上制备了三角形的 $\mathrm{BN}$ 晶畴及其薄膜. 通过使用锡䇴纸包裹原料的方法避免了残留原 料在 $\mathrm{BN}$ 表面的沉积. 当 $\mathrm{BN}$ 作为石墨烯场效应晶体管的介电层时, 基于石墨烯的场效应器件空穴与电子的迁移率分别为 10500 和 $4750 \mathrm{~cm}^{2} \mathrm{~V}^{-1} \mathrm{~s}^{-1}$, 明显优于在高温条件下制备的BN作为介电层的 石墨烯器件, 间接表明了该方法可得到高质量的BN. 另外, 本工作 也揭示了氮化硼的质量对石墨烯场效应器件的性能至关重要. 